Article

\title{
Differential Metabolic Reprogramming in Paenibacillus alvei-Primed Sorghum bicolor Seedlings in Response to Fusarium pseudograminearum Infection
}

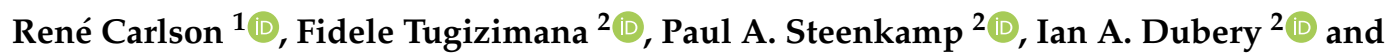 \\ Nico Labuschagne ${ }^{1, *}$ (1) \\ 1 Department of Plant and Soil Sciences, Faculty of Plant Pathology, University of Pretoria, Private Bag X20, \\ Hatfield, Pretoria 0028, South Africa \\ 2 Centre for Plant Metabolomics Research, Department of Biochemistry, Faculty of Science, \\ University of Johannesburg, P.O. Box 524, Auckland Park, Johannesburg 2006, South Africa \\ * Correspondence: nico.labuschagne@up.ac.za; Tel.: +27-827-807-849
}

Received: 25 April 2019; Accepted: 10 July 2019; Published: 23 July 2019

check for updates

\begin{abstract}
Metabolic changes in sorghum seedlings in response to Paenibacillus alvei (NAS-6G6)-induced systemic resistance against Fusarium pseudograminearum crown rot were investigated by means of untargeted ultra-high performance liquid chromatography-high definition mass spectrometry (UHPLC-HDMS). Treatment of seedlings with the plant growth-promoting rhizobacterium P. alvei at a concentration of $1 \times 10^{8}$ colony forming units $\mathrm{mL}^{-1}$ prior to inoculation with F. pseudograminearum lowered crown rot disease severity significantly at the highest inoculum dose of $1 \times 10^{6}$ spores $\mathrm{mL}^{-1}$. Intracellular metabolites were subsequently methanol-extracted from treated and untreated sorghum roots, stems and leaves at 1, 4 and 7 days post inoculation (d.p.i.) with F. pseudograminearum. The extracts were analysed on an UHPLC-HDMS platform, and the data chemometrically processed to determine metabolic profiles and signatures related to priming and induced resistance. Significant treatment-related differences in primary and secondary metabolism post inoculation with $F$. pseudograminearum were observed between $P$. alvei-primed versus naïve $S$. bicolor seedlings. The differential metabolic reprogramming in primed plants comprised of a quicker and/or enhanced upregulation of amino acid-, phytohormone-, phenylpropanoid-, flavonoid- and lipid metabolites in response to inoculation with $F$. pseudograminearum.
\end{abstract}

Keywords: crown rot; Fusarium pseudograminearum; induced systemic resistance; LC-MS; Paenibacillus alvei; PGPR; phytoalexin; priming; Sorghum bicolor

\section{Introduction}

As an evolutionary adaptation to survive a sessile existence, in addition to pre-existing physical and chemical barriers, plants have developed the ability to actively protect themselves against environmental stress through the synthesis of complex and ever-changing mixtures of defence-related metabolites [1]. These metabolites can either be synthesised locally or systemically as part of systemic acquired resistance (SAR) [2,3]. SAR has an associated fitness cost that has a negative impact on the plant's growth and development, but also indirectly impacts its microbiome [4]. It is hypothesised that in response to the negative ecological impact of SAR, evolutionary forces were directed towards the development of induced systemic resistance (ISR) [5]. During ISR, the plant is primed by beneficial microbes for an enhanced defensive response that only activates upon challenge with a stress, thus wasting no resources. 
During the early stages of priming, an increase in the sensitivity to the signalling hormones jasmonic acid or ethylene [6] facilitates the reprogramming of the plant's metabolome for enhanced defence [7-9]. Upon exposure to a stress, the synthesis of defence metabolites usually occur earlier and in higher quantities in primed compared with naïve plants [10], giving rise to the synthesis of secondary defence metabolites which include phenylpropanoids, terpenoids, polyketides and alkaloids [11].

Plant growth-promoting rhizobacteria (PGPR) are beneficial bacteria occurring in the root zone of plants, promoting plant growth directly through increased uptake of nutrients (biofertilisers), stimulation of plant growth through the production of phytohormones (biostimulants) or through the degradation of organic pollutants (rhizoremediators). PGPR also offer indirect plant growth promotion by protection against biotic- and abiotic stress (bioprotectants) [12]. PGPR can employ either one of these mechanisms or more than one, simultaneously [13-16]. In addition to this, PGPR are known to induce resistance systemically, giving rise to physical and/or chemical defence responses upon challenge with an external stress $[17,18]$.

Sorghum [Sorghum bicolor (L.) Moench] is an agricultural grain crop of significant importance for food security and sustainable livelihoods in developing countries [19]. In the current study, we investigated the protection offered by a PGPR, P. alvei, in mitigating the disease susceptibility of sorghum seedlings towards F. pseudograminearum, the causative agent of crown rot disease. During a time course study, we employed an untargeted ultra-high performance liquid chromatography-high definition mass spectrometry (UHPLC-HDMS)-based metabolomics approach to compare the adaptive metabolic changes that result in the altered metabolomes upon challenge with a biotic stress in primed versus naïve sorghum seedlings.

\section{Results}

\subsection{Plant Growth Parameters and Crown Rot Disease Severity}

The initial inoculum-dose study was aimed at optimising conditions for P. alvei-induced systemic resistance against crown rot. Crown rot disease severity increased significantly with increment in the inoculum dose (Table 1). Treatment with $P$. alvei caused a significant reduction in disease severity and an increase in root and shoot weights at the higher inoculum dose of $1 \times 10^{6}$ spores $\mathrm{mL}^{-1}$ (Table 1 ). Therefore, this inoculum dose was used in the time course study and it was found that disease severity increased as time progressed post inoculation (Figure 1A). However, the rate of disease progression was significantly lower in P. alvei-primed S. bicolor seedlings. This trend was correspondingly reflected in the fresh leaf- and root biomass of the seedlings (Figure 1B,C respectively).

To confirm that F. pseudograminearum was in fact the causal organism in both the inoculum doseand time course studies, isolations were made from crown rot lesions on the sorghum seedlings. Excised, surface-sterilised stem segments were plated onto rose bengal-glycerol-urea (RbGU) medium [20] and incubated under near-UV light to induce spore formation. F. pseudograminearum growth was identified morphologically by means of microscopy (Figure S1).

\subsection{Metabolomic Profiles of Paenibacillus alvei-Primed and Naïve Fusarium pseudograminearum Infected Sorghum Plants}

Visual inspection of the UHPLC-HDMS base peak intensity (BPI) chromatograms showed evidently differential peak population (presence, intensities) of P. alvei-primed- and naïve F. pseudograminearum infected S. bicolor seedlings versus the untreated controls. The chromatographically distinct BPI chromatograms of these three treatments for the electrospray ionisation (ESI)-positive data at 1 day post inoculation (d.p.i.) with F. pseudograminearum taken from root samples are shown in Figure 2 and those for stem- and leaf samples are provided in the supplementary material as Figures S2 and S3. These chromatographic differences reflect differential metabolite profiles (and composition) in samples derived from P. alvei-primed- and naïve F. pseudograminearum infected S. bicolor seedlings versus the untreated controls. 
Table 1. Effect of P. alvei alone and in combination with three dose levels of F. pseudograminearum on mean ${ }^{1}$ mass and disease severity of $S$. bicolor seedlings at 14 days post inoculation (d.p.i.).

\begin{tabular}{|c|c|c|c|c|c|c|c|c|c|c|c|c|c|}
\hline \multirow{2}{*}{$\begin{array}{c}\text { Treatment } \\
\begin{array}{c}\text { P. alvei } \\
\left(\mathrm{cfu} \mathrm{mL} \mathrm{m}^{-1}\right)^{2}\end{array}\end{array}$} & \multirow[b]{2}{*}{$\begin{array}{l}\text { F. pseudograminearum } \\
\text { (spores } \mathrm{mL}^{-1} \text { ) }\end{array}$} & \multicolumn{4}{|c|}{ Crown Rot } & \multicolumn{4}{|c|}{ Fresh Mass } & \multicolumn{4}{|c|}{ Dry Mass } \\
\hline & & Li-Rating & & $\begin{array}{c}\text { Isolations } \\
(\%)\end{array}$ & & $\begin{array}{l}\text { Leaves } \\
\text { (g) }\end{array}$ & & $\begin{array}{c}\text { Roots } \\
\text { (g) }\end{array}$ & & $\begin{array}{l}\text { Leaves } \\
\text { (g) }\end{array}$ & & $\begin{array}{l}\text { Roots } \\
\text { (g) }\end{array}$ & \\
\hline 0 & 0 & $0.00( \pm 0.00)$ & $\mathrm{e}$ & $0.00( \pm 0.00)$ & c & $0.68( \pm 0.16)$ & c & $0.73( \pm 0.30)$ & $\mathrm{a}$ & $0.14( \pm 0.07)$ & $\mathrm{a}$ & $0.04( \pm 0.01)$ & $\mathrm{ab}$ \\
\hline $1 \times 10^{8}$ & 0 & $0.00( \pm 0.00)$ & $\mathrm{e}$ & $0.00( \pm 0.00)$ & $\mathrm{c}$ & $0.75( \pm 0.21)$ & $\mathrm{ab}$ & $0.80( \pm 0.35)$ & $\mathrm{a}$ & $0.14( \pm 0.06)$ & $\mathrm{a}$ & $0.05( \pm 0.02)$ & $\mathrm{a}$ \\
\hline 0 & $1 \times 10^{2}$ & $1.14( \pm 1.03)$ & $\mathrm{cd}$ & $22.67( \pm 8.84)$ & $a b$ & $0.65( \pm 0.21)$ & c & $0.74( \pm 0.36)$ & $\mathrm{a}$ & $0.11( \pm 0.07)$ & $\mathrm{b}$ & $0.05( \pm 0.02)$ & $\mathrm{a}$ \\
\hline 0 & $1 \times 10^{4}$ & $1.90( \pm 0.96)$ & $\mathrm{b}$ & $24.00( \pm 9.66)$ & $a b$ & $0.67( \pm 0.18)$ & c & $0.68( \pm 0.28)$ & $\mathrm{a}$ & $0.10( \pm 0.05)$ & $\mathrm{b}$ & $0.05( \pm 0.02)$ & $\mathrm{a}$ \\
\hline 0 & $1 \times 10^{6}$ & $3.06( \pm 1.80)$ & $\mathrm{a}$ & $26.00( \pm 6.99)$ & $\mathrm{a}$ & $0.55( \pm 0.11)$ & d & $0.55( \pm 0.19)$ & $\mathrm{a}$ & $0.06( \pm 0.02)$ & c & $0.04( \pm 0.02)$ & $\mathrm{ab}$ \\
\hline $1 \times 10^{8}$ & $1 \times 10^{2}$ & $0.57( \pm 0.86)$ & de & $21.33( \pm 10.60)$ & $\mathrm{ab}$ & $0.58( \pm 0.19)$ & $\mathrm{d}$ & $0.65( \pm 0.32)$ & $\mathrm{a}$ & $0.10( \pm 0.07)$ & $\mathrm{b}$ & $0.03( \pm 0.00)$ & $\mathrm{b}$ \\
\hline $1 \times 10^{8}$ & $1 \times 10^{4}$ & $1.32( \pm 1.05)$ & $\mathrm{bc}$ & $21.00( \pm 11.01)$ & $a b$ & $0.70( \pm 0.24)$ & $\mathrm{bc}$ & $0.78( \pm 0.32)$ & $\mathrm{a}$ & $0.11( \pm 0.06)$ & $\mathrm{b}$ & $0.05( \pm 0.03)$ & $\mathrm{a}$ \\
\hline $1 \times 10^{8}$ & $1 \times 10^{6}$ & $1.67( \pm 1.35)$ & bc & $18.00( \pm 13.17)$ & $\mathrm{b}$ & $0.76( \pm 0.18)$ & $\mathrm{a}$ & $0.81( \pm 0.38)$ & $\mathrm{a}$ & $0.11( \pm 0.07)$ & $\mathrm{b}$ & $0.05( \pm 0.03)$ & a \\
\hline
\end{tabular}

${ }^{1}$ Means within columns followed by the same letter does not differ significantly according to Tukey's least significance determination (LSD) test at a significance level of $p<0.05$. Numbers

in parenthesis are the standard deviation from the mean. ${ }^{2}$ Colony forming units per millilitre. ${ }^{3}$ Disease severity rating calculated according to a '0-5 scale' based on lesion severity [21].

4 Percentage of the isolations made from the stem area that yielded growth on Fusarium-selective medium.
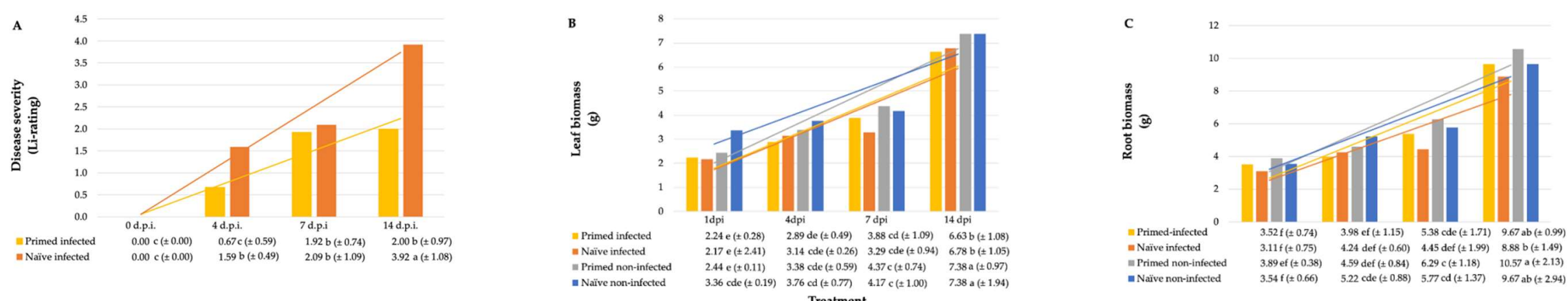

Figure 1. Effect of P. alvei alone and in combination with F. pseudograminearum on mean plant biomass and crown rot disease severity. (A) Crown rot disease severity and (B,C) leaf- and root biomass at 1, 4, 7 and 14 d.p.i. with F. pseudograminearum in P. alvei-primed versus naïve $S$. bicolor seedlings. Means followed by the same letter does not differ significantly according to Tukey's LSD test at a significance level of $p<0.05$. Numbers in parenthesis are the standard deviation from the mean. (A) Disease severity calculated according to a '0-5 scale' based on lesion severity [21]. Legend: Primed: Primed with P. alvei; Naïve (no priming); Infected: infected with F. pseudograminearum; Non-infected: not infected with F. pseudograminearum. 
In order to elucidate informative description of specific metabolic features related to these observed differential chromatographic profiles, data mining and comparative chemometric analyses were performed as described in the experimental section. Chemometric analyses employed included unsupervised methods, such as principal component analysis (PCA), hierarchical clustering analysis (HCA) and a supervised approach, namely orthogonal partial least square-discriminant analysis (OPLS-DA).

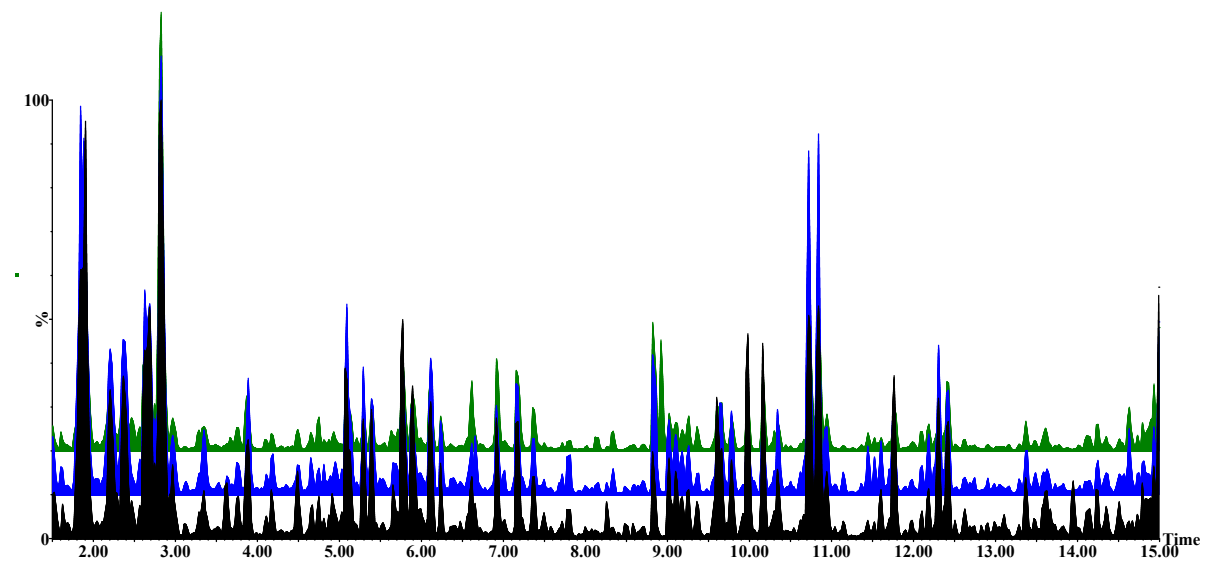

Figure 2. Untargeted ultra-high performance liquid chromatography-high definition mass spectrometry (UHPLC-HDMS) base peak intensity (BPI) chromatograms of electrospray ionisation (ESI)-positive data indicating the metabolomic profiles of untreated (black), naïve infected (blue) and primed infected (green) roots obtained at 1 d.p.i. with F. pseudograminearum.

The PCA and HCA computed for the root samples of the ESI-positive data are shown in Figure 3. The remaining models computed for the ESI-positive data (stem- and leaf samples) and ESI-negative data (root-, stem- and leaf-samples) are provided in the supplementary material as Figures S4-S8. The close clustering of the quality control (QC) samples in the PCA, indicate that the UHPLC-HDMS system was stable and that results were reproducible. The PCA models provide evidence for both treatment-related clustering (Figure 3A) and time-related clustering (Figure 3B).

The OPLS-DA computed for the root samples of the ESI-positive data is shown in Figure 4. Those for stem- and leaf samples are provided in the supplementary material as Figures S9 and S10 and a summary of the description and validation of all the computed OPLS-DA models is given in Table S1. All the OPLS-DA models used in this study were perfect binary classifiers and had no signs of possible overfitting, as indicated by cross-validation, and none of the permutated models $(n=100)$ performed better than the original models in separating classes, as typically shown by Figure 4B. For selection of 'variables', i.e., discriminating metabolite features with unique Rt $m / z$ values, OPLS-DA loadings S-plots (Figure 4C) were evaluated: these loading plots aid in identifying variables which differ between groups, i.e., the discriminating features. Variables that combine high model influence (covariation) with high reliability (correlation), i.e., variables at the far ends of the S-plot, are statistically relevant as potential discriminant variables to be selected [22]. To avoid bias in the selection of variables, the variable importance in projection (VIP)-plots were generated (Figure 4D) and only the variables (from S-plots) with VIP scores $>1.0$ were retained. Examples of variables that were significantly upregulated and identified as biomarkers in primed plants are highlighted in red. As mentioned in the experimental section, 41 statistically selected variables (from S-plots) were then annotated to MSI level-2 and are reported in Table 2, consisting of a total of 37 metabolites from the amino acid-, phytohormone-, flavonoid-, phenylpropanoid- and lipid metabolite class. To aid in the visualisation of the differential metabolic programming of primed plants compared with those left naïve, heatmap analysis was performed (Figure 5). In addition to this metabolic pathway- and Venn diagram- analyses are provided in the supplementary material as Figures S11 and S12. 

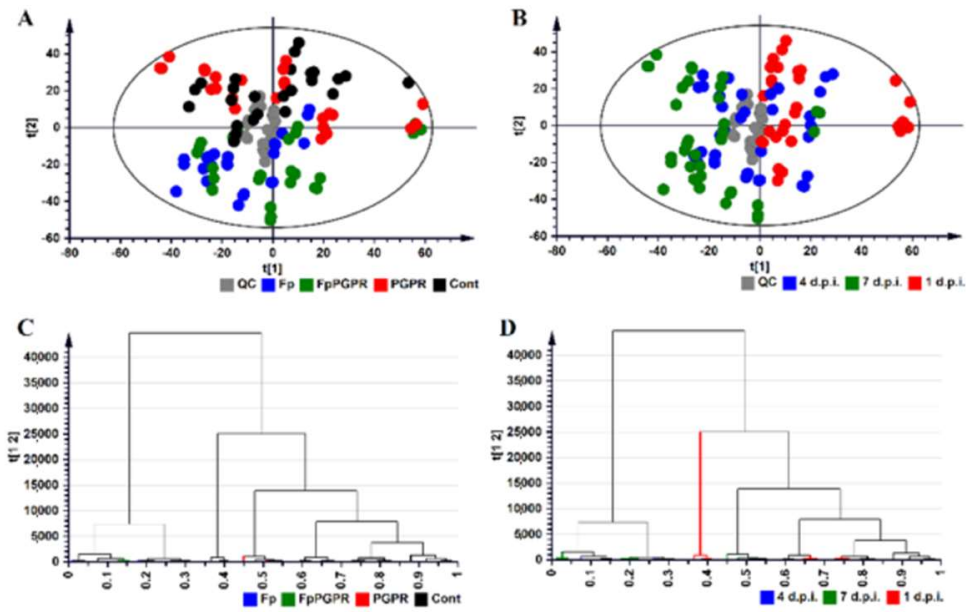

Figure 3. Principal component analysis (PCA) score/scatter plot of root samples computed from ESI-positive data representing the first two PCs of a 17-component PCA model. The model explains $74.3 \%$ variation in the Pareto-scaled data (the total amount of explained variation in $\mathrm{X} ; \mathrm{R}^{2} \mathrm{X}=0.743$ ) and $56.6 \%$ predicted variation according to cross-validation (the total amount of predicted variation; $\left.\mathrm{Q}^{2}=0.566\right)$. (A,B) represents the same PCA scores plot with (A) showing the treatment-related clustering and (B) showing the time-related clustering. (C,D) Hierarchical clustering analysis (HCA) dendrograms corresponding to (A,B). Legend: QC: Quality control (grey); Fp: Naïve plants inoculated with F. pseudograminearum (blue); FpPGPR: P. alvei-primed plants inoculated with F. pseudograminearum (green); PGPR: P. alvei-primed plants (red); Cont: Untreated plants (black); 1 d.p.i.: 1 d.p.i. with F. pseudograminearum (red); 4 d.p.i.: 4 d.p.i. with F. pseudograminearum (blue); 7 d.p.i.: 7 d.p.i. with F. pseudograminearum (green).
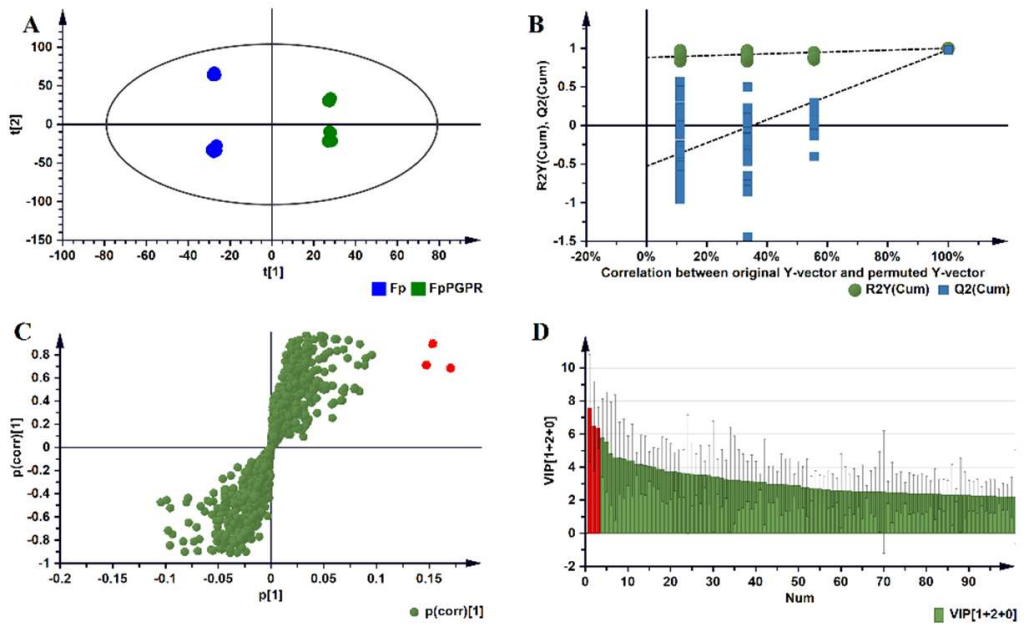

Figure 4. Orthogonal partial least square-discriminant analysis (OPLS-DA) modelling and variable/feature selection ESI-positive data (roots samples). (A) A typical PCA scores/scatter plot for the OPLS-DA model separating naïve (Fp) versus primed plants (FpPGPR) at 1 d.p.i. $\left(1+2+0\right.$ components, $R^{2} X=$ $0.630, \mathrm{Q}^{2}=0.980, \mathrm{CV}$-ANOVA, cross-validated analysis of variance, $p$-value $\left.<0.05\right)$. In the scores plot, it is evident that the two groups are clearly separated: naïve versus primed. (B) A typical response permutation test plot $(n=100)$ for the OPLS-DA model in $(A)$; the $R^{2}$ and $Q^{2}$ values of the permutated models correspond to $y$-axis intercepts: $R^{2}=(0.0,8.90)$ and $Q^{2}=(0.0,0.542)$. (C) An OPLS-DA loadings S-plot for the same model in (A); variables situated in the extreme end of the S-plot are statistically relevant and represent leading candidates as discriminating variables/features. (D) Variable importance for the projection (VIP) plot for the same model; pointing mathematically to the importance of each variable (feature) in contributing to group separation in the OPLS-DA model. (C,D) Examples of the variables that were significantly upregulated in primed plants are highlighted in red. 
Table 2. Summary of annotated metabolites upregulated in the roots (R), stems (S) and leaves (L) of F. pseudograminearum inoculated S. bicolor seedlings primed with P. alvei versus non-primed (naïve) seedlings. Discriminating metabolites were identified based on OPLS-DA S-plots.

\begin{tabular}{|c|c|c|c|c|c|c|c|c|c|c|c|c|c|}
\hline & \multirow{3}{*}{ Metabolite } & \multirow{3}{*}{ Heatmap Key } & \multirow{3}{*}{$\mathrm{m} / \mathrm{z}$} & \multirow{3}{*}{$\underset{(\min )}{\mathrm{Rt}}$} & \multirow{3}{*}{$\begin{array}{c}\text { ESI } \\
\text { Mode }\end{array}$} & \multirow{3}{*}{$\begin{array}{c}\text { Molecular } \\
\text { Formula/KEGG ID }\end{array}$} & \multirow{3}{*}{ Metabolite Class } & \multicolumn{6}{|c|}{ Metabolite Fold Change (fc) and $p$-Value of Primed $v s$. Naïve Seedlings } \\
\hline & & & & & & & & \multicolumn{2}{|c|}{1 d.p.i. } & \multicolumn{2}{|c|}{4 d.p.i. } & \multicolumn{2}{|c|}{7 d.p.i. } \\
\hline & & & & & & & & fc & $p$ & fc & $p$ & fc & p \\
\hline 1 & $\mathrm{~N}$-Carbamoylputrescine & Putrescine $\mathrm{R}$ & 176.08 & 4.66 & Pos & $\mathrm{C}_{5} \mathrm{H}_{13} \mathrm{~N}_{3} \mathrm{O} / \mathrm{C} 00436$ & Amino acid & 3.481 & $1.67 \times 10^{-5}$ & 5.401 & $2.00 \times 10^{-7}$ & 1.426 & $1.79 \times 10^{-2}$ \\
\hline 2 & L-2,4-Diaminobutanoate & DABA S & 163.05 & 5.59 & Pos & $\mathrm{C}_{4} \mathrm{H}_{10} \mathrm{~N}_{2} \mathrm{O}_{2} / \mathrm{C} 03283$ & Amino acid & 2.373 & $3.97 \times 10^{-2}$ & 0.287 & $4.30 \times 10^{-2}$ & 1.660 & $5.44 \times 10^{-1}$ \\
\hline 3 & THF-L-Glutamate & THF-L-Glu L & 595.19 & 1.65 & Neg & $\mathrm{C}_{24} \mathrm{H}_{30} \mathrm{~N}_{8} \mathrm{O}_{9} / \mathrm{C} 09332$ & Amino acid & 2.623 & $5.39 \times 10^{-2}$ & 2.770 & $1.12 \times 10^{-3}$ & 1.401 & $3.45 \times 10^{-1}$ \\
\hline 4 & Glutathione & Glu R & 326.12 & 2.93 & Pos & $\mathrm{C}_{10} \mathrm{H}_{17} \mathrm{~N}_{3} \mathrm{O}_{6} \mathrm{~S} / \mathrm{C} 00051$ & Amino acid & 5.562 & $4.22 \times 10^{-2}$ & 0.649 & $4.74 \times 10^{-1}$ & 1.045 & $9.54 \times 10^{-1}$ \\
\hline 5 & 2-(3-Carboxy-3-aminopropyl)-L-histidine & His $\mathrm{R}$ & 339.07 & 5.76 & Neg & $\mathrm{C}_{10} \mathrm{H}_{16} \mathrm{~N}_{4} \mathrm{O}_{4} / \mathrm{C} 04441$ & Amino acid & 2.845 & $1.21 \times 10^{-3}$ & 0.694 & $7.96 \times 10^{-2}$ & 0.643 & $8.00 \times 10^{-3}$ \\
\hline 6 & 2-(3-Carboxy-3-aminopropyl)-L-histidine & His S & 301.09 & 8.89 & Pos & $\mathrm{C}_{10} \mathrm{H}_{16} \mathrm{~N}_{4} \mathrm{O}_{4} / \mathrm{C} 04441$ & Amino acid & 2.362 & $2.08 \times 10^{-1}$ & 6.096 & $1.31 \times 10^{-2}$ & 0.600 & $3.27 \times 10^{-1}$ \\
\hline 7 & L-Lysine & Lys R & 213.09 & 2.14 & $\mathrm{Neg}$ & $\mathrm{C}_{6} \mathrm{H}_{14} \mathrm{~N}_{2} \mathrm{O}_{2} / \mathrm{C} 00047$ & Amino acid & 0.762 & $4.98 \times 10^{-1}$ & 4.140 & $1.05 \times 10^{-2}$ & 1.256 & $4.78 \times 10^{-1}$ \\
\hline 8 & N6,N6,N6-Trimethyl-L-lysine & Trimethyl Lys R & 313.09 & 3.60 & Neg & $\mathrm{C}_{9} \mathrm{H}_{20} \mathrm{~N}_{2} \mathrm{O}_{2} / \mathrm{C} 05546$ & Amino acid & 5.498 & $1.60 \times 10^{-3}$ & 4.479 & $1.70 \times 10^{-4}$ & 4.019 & $1.2 \times 10^{-2}$ \\
\hline 9 & L-phenylalanine & Phe R & 373.11 & 5.49 & Pos & $\mathrm{C}_{12} \mathrm{H}_{22} \mathrm{~N}_{4} \mathrm{O}_{7} / \mathrm{C} 00079$ & Amino acid & 32.594 & $3.39 \times 10^{-2}$ & 20.705 & $1.92 \times 10^{-2}$ & 3.180 & $1.54 \times 10^{-1}$ \\
\hline 10 & Guanosine 5'-diphosphate & GDP R & 675.88 & 1.15 & $\mathrm{Neg}$ & $\mathrm{C}_{10} \mathrm{H}_{17} \mathrm{~N}_{5} \mathrm{O}_{17} \mathrm{P}_{4} / \mathrm{C} 00035$ & Purine nucleoside & 129.214 & $1.56 \times 10^{-3}$ & 80.688 & $1.36 \times 10^{-4}$ & 48.241 & $6.52 \times 10^{-14}$ \\
\hline 11 & 8'-Hydroxyabscisate & ABAS & 301.11 & 9.67 & $\mathrm{Neg}$ & $\mathrm{C}_{15} \mathrm{H}_{20} \mathrm{O}_{5} / \mathrm{C} 15514$ & Phytohormone & 2.403 & $6.41 \times 10^{-2}$ & 2.502 & $7.68 \times 10^{-3}$ & 0.913 & $1.98 \times 10^{-2}$ \\
\hline 12 & Gibberellin A19 & GA19 R & 429.15 & 2.85 & $\mathrm{Neg}$ & $\mathrm{C}_{20} \mathrm{H}_{26} \mathrm{O}_{6} / \mathrm{C} 02034$ & Phytohormone & 2.055 & $2.94 \times 10^{-5}$ & 1.777 & $4.46 \times 10^{-2}$ & 1.345 & $3.92 \times 10^{-2}$ \\
\hline 13 & Gibberellin A8-catabolite & GA8 L & 383.11 & 8.87 & $\mathrm{Neg}$ & $\mathrm{C}_{19} \mathrm{H}_{22} \mathrm{O}_{7} / \mathrm{C} 11870$ & Phytohormone & 0.819 & $3.96 \times 10^{-1}$ & 1.822 & $4.08 \times 10^{-6}$ & 1.626 & $2.11 \times 10^{-3}$ \\
\hline 14 & Gibberellin A8-catabolite & GA8 R & 383.11 & 8.91 & $\mathrm{Neg}$ & $\mathrm{C}_{19} \mathrm{H}_{22} \mathrm{O}_{7} / \mathrm{C} 11870$ & Phytohormone & 1.781 & $1.62 \times 10^{-8}$ & 1.135 & $6.42 \times 10^{-1}$ & 2.585 & $2.33 \times 10^{-2}$ \\
\hline 15 & Salicin & Salicin R & 353.08 & 3.65 & $\mathrm{Neg}$ & $\mathrm{C}_{13} \mathrm{H}_{18} \mathrm{O}_{7} / \mathrm{C} 01451$ & Phytohormone & 14.920 & $2.96 \times 10^{-2}$ & 1.278 & $6.61 \times 10^{-1}$ & 4.174 & $1.14 \times 10^{-1}$ \\
\hline 16 & $\begin{array}{l}\text { (-)-11-Hydroxy-9,10-dihydrojasmonic acid } \\
\text { 11-beta-D-glucoside }\end{array}$ & JAR R & 435.19 & 9.22 & $\mathrm{Neg}$ & $\mathrm{C}_{18} \mathrm{H}_{28} \mathrm{O}_{9} / \mathrm{C} 21385$ & Phytohormone & 27.667 & $1.05 \times 10^{-3}$ & 78.994 & $9.42 \times 10^{-3}$ & 4.373 & $2.32 \times 10^{-2}$ \\
\hline 17 & (-)-Jasmonoyl-L-valine & JA-Val R & 368.16 & 5.49 & Pos & $\mathrm{C}_{17} \mathrm{H}_{27} \mathrm{NO}_{4} / \mathrm{C} 21509$ & Phytohormone & 25.519 & $2.37 \times 10^{-2}$ & 3.025 & $2.40 \times 10^{-1}$ & 3.127 & $2.64 \times 10^{-1}$ \\
\hline 18 & Dihydrozeatin & Zeatin R & 242.10 & 6.14 & $\mathrm{Neg}$ & $\mathrm{C}_{10} \mathrm{H}_{15} \mathrm{~N}_{5} \mathrm{O} / \mathrm{C} 02029$ & Phytohormone & 96.477 & $2.10 \times 10^{-3}$ & 15.249 & $1.08 \times 10^{-3}$ & 2.199 & $2.04 \times 10^{-1}$ \\
\hline 19 & (-)-Epicatechin & Epicatechin S & 289.07 & 3.50 & $\mathrm{Neg}$ & $\mathrm{C}_{15} \mathrm{H}_{14} \mathrm{O}_{6} / \mathrm{C} 09727$ & Phenylpropanoid & 1.455 & $7.08 \times 10^{-3}$ & 2.749 & $1.50 \times 10^{-2}$ & 1.299 & $4.98 \times 10^{-1}$ \\
\hline 20 & (-)-Hesperetin & Hesperetin S & 301.07 & 8.28 & Neg & $\mathrm{C}_{16} \mathrm{H}_{14} \mathrm{O}_{6} / \mathrm{C} 01709$ & Phenylpropanoid & 3.301 & $1.81 \times 10^{-2}$ & 3.799 & $4.39 \times 10^{-4}$ & 0.697 & $4.01 \times 10^{-1}$ \\
\hline 21 & 4-Coumaroylshikimate & CoumaroylSA R & 336.11 & 4.70 & $\mathrm{Neg}$ & $\mathrm{C}_{16} \mathrm{H}_{16} \mathrm{O}_{7} / \mathrm{C} 02947$ & Phenylpropanoid & 4.580 & $6.11 \times 10^{-6}$ & 5.575 & $5.88 \times 10^{-6}$ & 1.760 & $5.16 \times 10^{-6}$ \\
\hline 22 & 7-O-D-Glucosyl-apigenin & Apigenin S & 477.10 & 5.95 & $\mathrm{Neg}$ & $\mathrm{C}_{21} \mathrm{H}_{20} \mathrm{O}_{10} / \mathrm{C} 04608$ & Phenylpropanoid & 0.804 & $5.84 \times 10^{-1}$ & 1.935 & $1.77 \times 10^{-1}$ & 9.563 & $3.73 \times 10^{-4}$ \\
\hline 23 & 8-C-Glucosylnaringenin & Naringenin R & 433.11 & 6.00 & $\mathrm{Neg}$ & $\mathrm{C}_{21} \mathrm{H}_{22} \mathrm{O}_{10} / \mathrm{C} 16492$ & Phenylpropanoid & 0.631 & $8.01 \times 10^{-2}$ & 2.588 & $7.39 \times 10^{-3}$ & 1.683 & $6.31 \times 10^{-2}$ \\
\hline 24 & Kaempferol 3-O-D-Glucosylgalactoside & Kaempferol L & 609.15 & 5.44 & $\mathrm{Neg}$ & $\mathrm{C}_{27} \mathrm{H}_{30} \mathrm{O}_{16} / \mathrm{C} 16490$ & Phenylpropanoid & 0.877 & $8.41 \times 10^{-1}$ & 5.543 & $3.2 \times 10^{-3}$ & 1.650 & $3.54 \times 10^{-1}$ \\
\hline 25 & Leucocyanidin & Leucocyanidin $\mathrm{R}$ & 322.09 & 4.67 & $\mathrm{Neg}$ & $\mathrm{C}_{15} \mathrm{H}_{14} \mathrm{O}_{7} / \mathrm{C} 05906$ & Phenylpropanoid & 6.203 & $3.03 \times 10^{-10}$ & 2.208 & $6.51 \times 10^{-3}$ & 1.622 & $8.90 \mathrm{E} \times 10^{-2}$ \\
\hline 26 & Neohesperidin & Neohesperidin R & 609.18 & 4.91 & $\mathrm{Neg}$ & $\mathrm{C}_{28} \mathrm{H}_{34} \mathrm{O}_{15} / \mathrm{C} 09806$ & Phenylpropanoid & 0.968 & $6.11 \times 10^{-1}$ & 2.025 & $6.52 \times 10^{-5}$ & 2.579 & $3.58 \times 10^{-4}$ \\
\hline 27 & 5-O-Caffeoylshikimic acid & Shikimic acid $\mathrm{R}$ & 352.10 & 4.01 & $\mathrm{Neg}$ & $\mathrm{C}_{16} \mathrm{H}_{16} \mathrm{O}_{8} / \mathrm{C} 10434$ & Phenylpropanoid & 743.006 & $9.33 \times 10^{-8}$ & 11.814 & $9.87 \times 10^{-5}$ & 4.179 & $4.54 \times 10^{-5}$ \\
\hline 28 & Caffeic acid 3-glucoside & Caffeic acid R & 341.09 & 6.25 & $\mathrm{Neg}$ & $\mathrm{C}_{15} \mathrm{H}_{18} \mathrm{O}_{9} / \mathrm{C} 10431$ & Phenylpropanoid & 6.106 & $5.83 \times 10^{-2}$ & 6.521 & $5.20 \times 10^{-2}$ & 4.845 & $2.63 \times 10^{-2}$ \\
\hline 29 & Caffeoylquinate & Caffeoylquinate $\mathrm{R}$ & 353.09 & 2.62 & $\mathrm{Neg}$ & $\mathrm{C}_{16} \mathrm{H}_{17} \mathrm{KO}_{9} / \mathrm{C} 00852$ & Phenylpropanoid & 7.071 & $2.65 \times 10^{-2}$ & 4.685 & $1.35 \times 10^{-2}$ & 5.660 & $8.72 \times 10^{-2}$ \\
\hline 30 & Trans-D-Glucosyl-2-hydroxycinnamate & Cinnamic acid L & 325.09 & 3.71 & $\mathrm{Neg}$ & $\mathrm{C}_{15} \mathrm{H}_{18} \mathrm{O}_{8} / \mathrm{C} 05158$ & Phenylpropanoid & 1.517 & $3.37 \times 10^{-1}$ & 3.486 & $4.97 \times 10^{-2}$ & 1.485 & $3.20 \times 10^{-1}$ \\
\hline
\end{tabular}


Table 2. Cont

\begin{tabular}{|c|c|c|c|c|c|c|c|c|c|c|c|c|c|}
\hline & \multirow{3}{*}{ Metabolite } & \multirow{3}{*}{ Heatmap Key } & \multirow{3}{*}{$m / z$} & \multirow{3}{*}{$\begin{array}{c}\mathrm{Rt} \\
(\mathrm{min})\end{array}$} & \multirow{3}{*}{$\begin{array}{l}\text { ESI } \\
\text { Mode }\end{array}$} & \multirow{3}{*}{$\begin{array}{c}\text { Molecular } \\
\text { Formula/KEGG ID }\end{array}$} & \multirow{3}{*}{ Metabolite Class } & \multicolumn{6}{|c|}{ Metabolite Fold Change (fc) and $p$-Value of Primed $v s$. Naïve Seedlings } \\
\hline & & & & & & & & \multicolumn{2}{|c|}{1 d.p.i. } & \multicolumn{2}{|c|}{$\underline{4 \text { d.p.i. }}$} & \multicolumn{2}{|c|}{7 d.p.i. } \\
\hline & & & & & & & & fc & $p$ & fc & $p$ & fc & $p$ \\
\hline 31 & Trans-D-Glucosyl-2-hydroxycinnamate & Cinnamic acid $\mathrm{R}$ & 325.09 & 3.75 & $\mathrm{Neg}$ & $\mathrm{C}_{15} \mathrm{H}_{18} \mathrm{O}_{8} / \mathrm{C} 05158$ & Phenylpropanoid & 1.907 & $1.33 \times 10^{-1}$ & 2.338 & $2.18 \times 10^{-2}$ & 1.224 & $4.75 \times 10^{-1}$ \\
\hline 32 & Trans-D-Glucosyl-2-hydroxycinnamate & Cinnamic acid S & 325.09 & 6.94 & Neg & $\mathrm{C}_{15} \mathrm{H}_{18} \mathrm{O}_{8} / \mathrm{C} 05158$ & Phenylpropanoid & 1.644 & $9.05 \times 10^{-3}$ & 2.238 & $4.44 \times 10^{-5}$ & 1.634 & $1.73 \times 10^{-1}$ \\
\hline 33 & (6Z,9Z,12Z)-Octadecatrienoic acid & $\gamma$-linolenic acid $\mathrm{R}$ & 345.20 & 1.92 & Neg & $\mathrm{C}_{18} \mathrm{H}_{30} \mathrm{O}_{2} / \mathrm{C} 06426$ & Lipid & 3.122 & $1.18 \times 10^{-8}$ & 3.157 & $4.37 \times 10^{-8}$ & 2.392 & $2.61 \times 10^{-9}$ \\
\hline 34 & $\begin{array}{c}\text { 9-Hydroperoxy-12,13- } \\
\text { epoxy-10-octadecenoic acid }\end{array}$ & OA L & 371.18 & 5.27 & Neg & $\mathrm{C}_{18} \mathrm{H}_{32} \mathrm{O}_{4} / \mathrm{C} 08368$ & Lipid & 445.872 & $2.44 \times 10^{-14}$ & 691.790 & $2.44 \times 10^{-14}$ & 818.658 & $2.44 \times 10^{-14}$ \\
\hline 35 & 12,13-Epoxy-9-hydroxy-10-octadecenoate & Oleic acid R & 361.20 & 1.14 & $\mathrm{Neg}$ & $\mathrm{C}_{18} \mathrm{H}_{32} \mathrm{O}_{4} / \mathrm{C} 14832$ & Lipid & 2.041 & $2.15 \times 10^{-8}$ & 1.459 & $1.17 \times 10^{-5}$ & 1.322 & $1.04 \times 10^{-3}$ \\
\hline 36 & $\begin{array}{l}\text { Methyl 9-hydroperoxy-10,12,13,15- } \\
\text { bisepidioxy-16E-octadecenoate }\end{array}$ & Oleic acid L & 461.14 & 1.60 & $\mathrm{Neg}$ & $\mathrm{C}_{19} \mathrm{H}_{32} \mathrm{O}_{8 /} \mathrm{C} 14832$ & Lipid & 3.756 & $4.42 \times 10^{-3}$ & 0.419 & $2.13 \times 10^{-1}$ & 0.968 & $9.51 \times 10^{-1}$ \\
\hline 37 & 18-Fluoro-octadecanoic acid & Fluoro-OA R & 347.23 & 1.57 & Pos & $\mathrm{C}_{18} \mathrm{H}_{35} \mathrm{FO}_{2} / \mathrm{C} 01530$ & Lipid & 76.144 & $1.20 \times 10^{-2}$ & 176.354 & $7.82 \times 10^{-9}$ & 474.046 & $6.61 \times 10^{-4}$ \\
\hline 38 & (4E,8E,10E-d18:3) Sphingosine & Sphingosine $\mathrm{R}$ & 318.24 & 1.02 & Pos & $\mathrm{C}_{18} \mathrm{H}_{33} \mathrm{NO}_{2} / \mathrm{C} 00319$ & Lipid & 9.621 & $2.96 \times 10^{-1}$ & 288.427 & $2.68 \times 10^{-2}$ & 111.911 & $2.14 \times 10^{-1}$ \\
\hline 39 & $3,4,3^{\prime}, 4^{\prime}$-tetrahydrospirilloxanthin & Spirilloxanthin $\mathrm{R}$ & 673.42 & 1.21 & $\mathrm{Neg}$ & $\mathrm{C}_{42} \mathrm{H}_{64} \mathrm{O}_{2} / \mathrm{C} 15888$ & Lipid & 9.271 & $2.34 \times 10^{-2}$ & 3.857 & $5.20 \times 10^{-3}$ & 1.329 & $9.69 \times 10^{-2}$ \\
\hline 40 & 5-Formiminotetrahydrofolate & Formimino-THF L & 516.14 & 5.12 & $\mathrm{Neg}$ & $\mathrm{C}_{20} \mathrm{H}_{24} \mathrm{~N}_{8} \mathrm{O}_{6} / \mathrm{C} 00664$ & Vitamin & 3.140 & $3.34 \times 10^{-3}$ & 1.363 & $3.79 \times 10^{-1}$ & 2.074 & $6.33 \times 10^{-2}$ \\
\hline 41 & Dhurrin & Dhurrin S & 310.09 & 2.82 & $\mathrm{Neg}$ & $\mathrm{C}_{14} \mathrm{H}_{17} \mathrm{NO}_{7} / \mathrm{C} 05143$ & Cyanogenic glucoside & 2.186 & $5.67 \times 10^{-4}$ & 4.514 & $1.96 \times 10^{-3}$ & 2.545 & $1.04 \times 10^{-1}$ \\
\hline
\end{tabular}




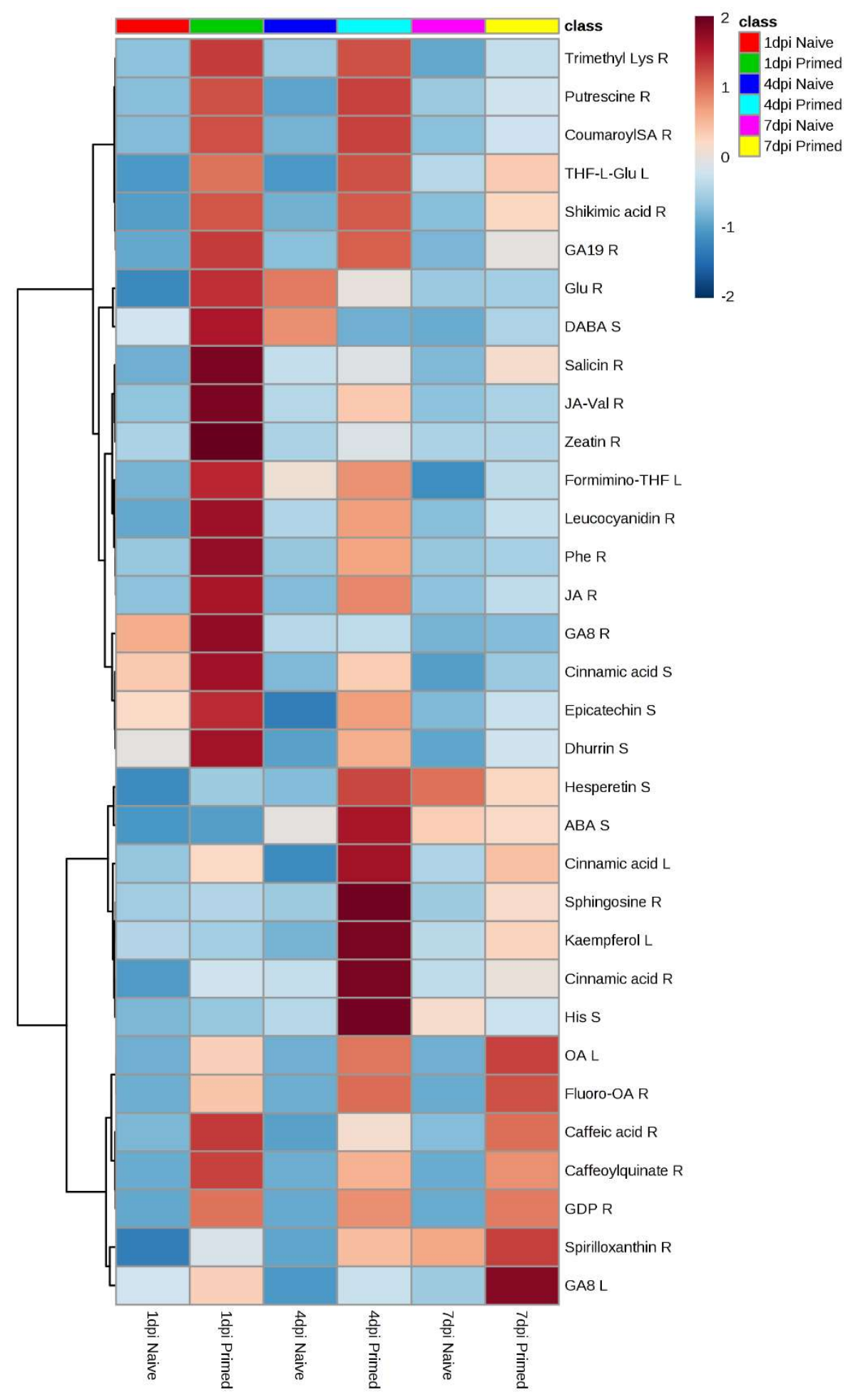

Figure 5. Heatmap showing the top 33 metabolites (abbreviations listed in Table 2 under the heading "Heatmap key") upregulated in roots (R), stems (S) and leaves (L) of P. alvei-primed and naïve S. bicolor seedlings at 1,4 and 7 d.p.i. with F. pseudograminearum

\section{Discussion}

During the ISR response, specific consecutive steps are necessary for a successful tripartite interaction, namely (1) an initial plant-microbe recognition, (2) the activation of downstream signal transduction pathways during priming and, finally, (3) the defence response upon challenge with a pathogen [23]. In 
the current study a similar interaction between P. alvei, S. bicolor and F. pseudograminearum gave rise to ISR. Priming S. bicolor seedlings with $P$. alvei resulted in an early, enhanced upregulation of both primary and secondary metabolism upon inoculation with F. pseudograminearum as shown in the pathway analysis (provided in the supplementary material as Figure S11). Pathways involved in phytohormone-, amino acid- and flavonoid- metabolism were affected.

In the current study, the early upregulation of jasmonic acid (JA) $(28 \times$ change, Table 2$)$ in P. alvei-primed roots at 1 d.p.i. with F. pseudograminearum supports previous findings of its requirement in the initial signal transduction during ISR, giving rise to a reprogramming of the plant's metabolome for enhanced defence. Although JA or ethylene is required in ISR, other phytohormones also play a role in signal transduction for enhanced defence $[23,24]$. In the current study zeatin $(96 \times$ change), salicin $(15 \times$ change $)$ and gibberellin $(2 \times$ change $)$ were upregulated in the roots of primed S. bicolor seedlings (Table 2). These phytohormones play an important role in plant growth, but also in the protection against stress. When secreted by rhizobacteria for instance, these phytohormones also act as signalling factors that elicit plant defences $[25,26]$.

In addition to the upregulation of phytohormones in primed plants, the changes in primary metabolism also included the group of amino acids, which serve as building blocks for secondary metabolism. Priming thus preconditions the plant for enhanced defence by elevating the amino acid levels that are at the disposal of the plant. In the current study, amino acids and derivatives were upregulated as early as 1 d.p.i. with F. pseudograminearum, including phenylalanine $(33 \times$ change), glutathione $(6 \times$ change) $)$ lysine $(6 \times$ change), putrescine $(4 \times$ change $)$ and histidine $(3 \times$ change $)($ Table 2$)$. Aromatic amino acids such as phenylalanine, that derive from the shikimate pathway, enable the early upregulation of the phenylpropanoid pathway and resultant phenylpropanoid defence metabolites, including phytoalexins, cell wall reinforcements and wound response $[27,28]$. Glutathione is a tripeptide thiol (consisting of glycine, glutamate and cysteine) that has powerful antioxidant capacity thus offering protection against oxidative stress [29]. It protects macromolecules such as lipids, proteins and DNA from free radical damage through a process of glutathiolation; it acts as a proton donor in the presence of free radicals and plays a role in the production of other antioxidant such as ascorbate.

Secondary metabolites that possess antifungal properties against the genus Fusarium [30] that were significantly upregulated in primed versus naïve $S$. bicolor seedlings included shikimic acid, $(743 \times$ change at 1 d.p.i. with F. pseudograminearum, (Table 2), the precursor of phenylpropanoids that include flavonoids and cinnamate derivatives. Flavonoids that were found to be upregulated included: epicatechin $(2 \times$ change at 1 d.p.i.) [31-33], hesperetin ( $3 \times$ change at 1 d.p.i.) [34,35], apigenin $(10 \times$ change at 7 d.p.i.) $[33,36,37]$, naringenin $(3 \times$ change at 4 d.p.i. $)[33,36]$, kaempferol $(6 \times$ change at 4 d.p.i.) [33,36], leucocyanidin $(6 \times$ change at 1 d.p.i.) [32] and neohesperidin $(2 \times$ change at 4 d.p.i.) [34] (Table 2). Hydroxycinnamic acids and derivatives found to be upregulated included: caffeic acid ( $5 \times$ change at 7 d.p.i.) [38], coumaroylshikimate [39], caffeoylquinate $(7 \times$ change at 1 d.p.i.) [30] and cinnamic acid $(2 \times$ change1 d.p.i.) $[33,38]$ (Table 2$)$.

The group of fatty acids, that were significantly upregulated earlier in primed versus naïve S. bicolor seedlings, were $\gamma$-linolenic acid $(3 \times$ change $)$, octadecenoic acid $(7 \times$ change $)$ and oleic acid $(446 \times$ change). These fatty acids are found in higher levels in grain varieties that has resistance to Fusarium-diseases [40]. Other lipids that were upregulated included sphingosine $(288 \times$ change at 4 d.p.i.) and spirilloxanthin (a carotenoid ether, $-9 \times$ change at 1 d.p.i.) (Table 2). Sphingosine is a cell membrane fatty acid that plays an important role in signalling and programmed cell death [41,42].

The vitamin folic acid was also upregulated in primed plants. Folate, like most vitamins, plays an important role in protecting plants against stress, as it possesses strong antioxidant potential $[43,44]$. The cyanogenic glucoside dhurrin was significantly upregulated in primed S. bicolor seedlings. Cyanogenic glucosides are known to liberate $\mathrm{HCN}$, which is fungitoxic [45]. The occurrence of dhurrin in stems of primed plants infected with F. pseudograminearum, indicate that it might have played a role in suppressing Fusarium infection [46]. 
Sorghum contains large quantities of phenols and antioxidants, crucial in active defence against biotic [47] and abiotic stresses [48]. In a study done by Tugizimana et al. [47], the defence-related metabolic reprogramming in S. bicolor in response to Colletotrichum sublineolum inoculation implicated the activation of both the early phenylpropanoid and flavonoid metabolic pathways as part of the defence response. In the current study, similar defence-related metabolites were upregulated in P. alvei-primed S. bicolor plants in response to F. pseudograminearum inoculation during ISR. These results provide evidence for significant overlap in the metabolic reprogramming between non-ISR and ISR mediated defence in S. bicolor, including the defence metabolites such as apigenin, naringenin, kaempferol, other flavonoid conjugates and hydroxycinnamate conjugates such as caffeoylquinate. Moreover, when the metabolic defence-related reprogramming of P. alvei-primed S. bicolor plants is compared to non-primed plants, it is clear that priming resulted in a quicker and/or enhanced upregulation of amino acid-, phytohormone-, flavonoid-, phenylpropanoid- and lipid metabolites in response to inoculation with F. pseudograminearum (Figure 5).

\section{Materials and Methods}

\subsection{Greenhouse Assessment of Induced Systemic Resistance}

\subsubsection{Inoculum Preparation}

PGPR: A PGPR strain (Paenibacillus alvei NAS-6G6) that has previously shown plant growth enhancement and biocontrol activity on cereal crops [49] was obtained from the PGPR collection of the University of Pretoria (Pretoria, South Africa). The bacterial strain was maintained at $-72{ }^{\circ} \mathrm{C}$ on Microbeads ${ }^{\circledR}$ (Davies Diagnostics, Randburg, South Africa). The strain was streaked onto Nutrient agar and a $1 \mathrm{w}$ old culture was inoculated into Nutrient broth and incubated in a rotary shaker at $25^{\circ} \mathrm{C}$ and $150 \mathrm{rpm}$ for $48 \mathrm{~h}$. The bacterial suspension was subsequently centrifuged in $50 \mathrm{~mL}$ capacity sterile plastic tubes at $2000 \mathrm{rpm}$ for $10 \mathrm{~min}$. The resulting pellet was re-suspended in quarter strength sterile Ringer's solution to give a final concentration of $10^{8} \mathrm{cfu} \mathrm{mL}^{-1}$.

Fungal pathogen: Fusarium pseudograminearum (strain M7816N) was obtained from Dr. Sandra Lamprecht at the Agricultural Research Council Plant Protection Research Institute, Stellenbosch, South Africa. The strain was maintained in culture on filter paper at $5{ }^{\circ} \mathrm{C}$ and, when needed, plated onto half strength potato dextrose agar (PDA) [50]. Five $5 \mathrm{~mm}$ diameter discs were subsequently taken from the edge of a $72 \mathrm{~h}$ culture and inoculated into $500 \mathrm{~mL}$ mungbean liquor medium [51].

\subsubsection{Sorghum Cultivation}

Sorghum bicolor seed (cultivar Sweet NS 5655) was obtained from Advance Seed (Krugersdorp, South Africa). The seeds were sterilised successively in $70 \%$ ethanol ( $5 \mathrm{~min}$ ), $1 \%$ sodium hypochlorite ( $1 \mathrm{~min}$ ) and rinsed five times with sterile $\mathrm{dH}_{2} \mathrm{O}$. The seeds were subsequently transferred to Petri dishes containing filter paper moistened with sterile $\mathrm{dH}_{2} \mathrm{O}$ and allowed to germinate for $48 \mathrm{~h}$ at $25^{\circ} \mathrm{C}$. The germinating seeds were inspected daily for any bacterial and fungal growth and contaminated seedlings were discarded. The $S$. bicolor germlings were directly planted into plastic seedling trays filled with washed, autoclaved $\left(120^{\circ} \mathrm{C}\right.$ for $\left.20 \mathrm{~min}\right)$, pure silica sand. The trays consisted of $30 \times 50 \mathrm{~mL}$ cells per tray and were sterilised with $10 \%$ sodium hypochlorite. The plants were watered every second day with sterilised $\mathrm{dH}_{2} \mathrm{O}$ to field capacity. The seedlings were fertilised once a week with a general water soluble fertiliser (Multifeed ${ }^{\circledR}$, Nulandis, Kempton Park, South Africa). No pesticides or fungicides were needed. The greenhouse temperature was maintained at between $20^{\circ} \mathrm{C}$ and $30^{\circ} \mathrm{C}$ and the relative humidity fluctuated between $40 \%$ and $60 \%$. At harvest, the fresh and dry weights of both roots and shoots were measured and samples for metabolomic analysis were taken as detailed under 4.2.1. The experimental design consisted of three independent biological repeats. 


\subsubsection{Treatment (Priming) with Paenibacillus alvei and Inoculation with Fusarium pseudograminearum}

PGPR: Seedlings were treated with P. alvei $1 \mathrm{~d}$ prior to inoculation with F. pseudograminearum [52]. The sand around each individual seedling was drenched with $1 \mathrm{~mL}$ of the $P$. alvei cell suspension at $10^{8} \mathrm{cfu} \mathrm{mL}^{-1}$.

Fungal pathogen: One day after treatment of sorghum seedlings with $P$. alvei, the plants were inoculated with the fungal pathogen. To ensure spatial separation from $P$. alvei in the root zone, a small piece $(30 \times 15 \mathrm{~mm})$ of sterilised absorbent cotton wool was wrapped around the base of each stem as shown in Figure 6, at ca. $1 \mathrm{~cm}$ above the surface of the sand and held in place by masking tape (15 mm wide). The plants were pipette-inoculated with the fungal pathogen by adding $500 \mu \mathrm{L}$ of a conidial suspension of F. pseudograminearum onto the cotton wool in a similar fashion as previously described by Mitter et al. 2006 [53]. Final spore suspension concentrations of $1 \times 10^{2}, 1 \times 10^{4}$ and $1 \times 10^{6}$ spores $\mathrm{mL}^{-1}$ were used for inoculation $[54,55]$. Control treatments received $500 \mu \mathrm{L}$ of sterile mungbean liquor medium.
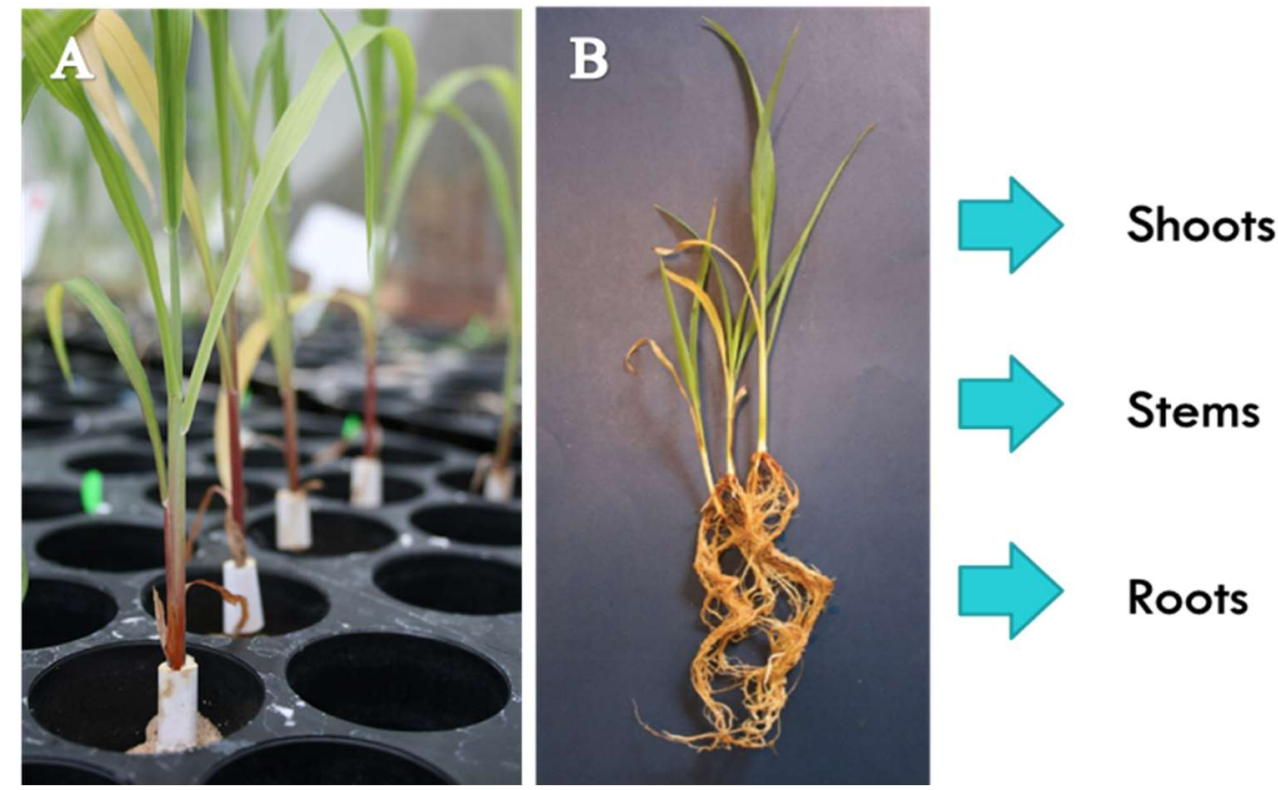

Figure 6. (A) Sterilised, absorbent cotton wool wrapped around the stems of S. bicolor seedlings to enable pipette-inoculation with a F. pseudograminearum spore suspensions. (B) Samples were taken from roots (R), stems (S) and shoots (leaves, L) of F. pseudograminearum inoculated, P. alvei amended, primed (P. alvei amended and F. pseudograminearum inoculated) and untreated (control) S. bicolor seedlings.

\subsubsection{Crown Rot Disease Severity and Confirmation of Koch's Postulates}

Li-rating: At harvest, disease severity was assessed at 1, 4, 7 and 14 d.p.i. according to the Wildermuth and McNamara '0-5 scale' as modified by Li et al. [21], where $0=$ no obvious symptom; $1=$ visible necrotic lesion on coleoptile or first leaf sheath; 2 = the first leaf sheath and below sub-crown internode partially necrotic; 3 = the second leaf sheath and the below sub-crown internode completely necrotic with up to $50 \%$ reduction in seedling height; $4=$ the third leaf or leaf sheath and the below sub-crown internode partially or completely necrotic with more than $50 \%$ reduction of seedling height; 5 = whole plant severely to completely necrotic.

Fungal isolation: In order to confirm Koch's postulates, segments from the crown area from both infected and non-infected plants were excised and the surface was sterilised with $0.5 \%$ sodium hypochlorite for $2 \mathrm{~min}$ and rinsed five times with sterile water. These segments were aseptically plated in triplicate on rose bengal-glycerol-urea (RbGU) medium [20]. The plates were incubated at $27 \pm 1{ }^{\circ} \mathrm{C}$ for $7 \mathrm{~d}$ and the resulting fungal colonies were examined microscopically. 


\subsubsection{Statistical Analyses of Growth Parameters and Disease Assessments}

Data were subjected to analysis of variance and means were compared using Tukey's least significance determination (LSD) test at a significance level of $p=0.05$.

\subsection{Metabolite Profiling}

\subsubsection{Sample Collection}

At harvest, fresh samples were collected from roots, stems and leaves of (1) F. pseudograminearuminoculated, (2) P. alvei-amended, (3) primed (P. alvei amended and F. pseudograminearum inoculated) and (4) untreated (control) S. bicolor seedlings (Figure 6). Samples were consecutively taken from each seedling, first from leaves, then stems and lastly roots. Root samples were carefully removed from the growing medium (pure silica sand) by adding sterilised $\mathrm{dH}_{2} \mathrm{O}$ to each pot (up to field capacity) to loosen the roots. The roots were then carefully washed with sterilised $\mathrm{dH}_{2} \mathrm{O}$ and dried with tissue paper. Samples were immediately weighed, placed in $50 \mathrm{~mL}$ centrifuge tubes, frozen with liquid nitrogen and kept at $-72{ }^{\circ} \mathrm{C}$ until time of metabolite extraction.

Right before extraction, the frozen samples were carefully crushed to a powder by making use of a clean spatula and kept frozen by adding liquid nitrogen as needed. One gram of the crushed sample was then carefully transferred to a sterile $50 \mathrm{~mL}$ centrifuge tube. The extraction process followed immediately thereafter making sure that the sample remained frozen up to this point.

\subsubsection{Metabolite Extraction}

Intracellular metabolites were extracted with $80 \%$ methanol [1:10 (w/v)] from roots, stems and leaves of (1) F. pseudograminearum-inoculated, (2) P. alvei-amended, (3) primed (P. alvei amended and F. pseudograminearum inoculated) and (4) untreated (control) S. bicolor seedlings (Figure 6) at 1 , 4 and 7 d.p.i. with F. pseudograminearum which coincided with 2-, 5- and $8 \mathrm{~d}$ post treatment with P. alvei, respectively (it is important to note that the root samples also contained P. alvei metabolites and stem samples also contained F. pseudograminearum metabolites). The $80 \%$ methanol mixture was homogenised using an Ultra Turrax homogeniser. The samples were subsequently centrifuged for $20 \mathrm{~min}$ at $5100 \mathrm{rpm}$ at $4^{\circ} \mathrm{C}$. Supernatants were removed and evaporated under vacuum by using a rotary evaporator at $55^{\circ} \mathrm{C}$ to a final volume of approximately $1 \mathrm{~mL}$ and transferred to Eppendorf tubes where it was dried in a vacuum centrifuge at $40^{\circ} \mathrm{C}$ for $6 \mathrm{~h}$ to complete dryness. The dried samples were subsequently resuspended to a final volume of $500 \mu \mathrm{L}$ (80\% aqueous LC-grade methanol and $20 \%$ ultrapure water) and filtered through $0.22 \mu \mathrm{m}$ nylon syringe filters (Anatech, Randburg, South Africa) into high performance liquid chromatography glass vials fitted with $500 \mu \mathrm{L}$ inserts and stored at $-20^{\circ} \mathrm{C}$. For quality control $(\mathrm{QC})$ purposes, pooled samples were prepared by pipetting and mixing aliquots of equal volume from all samples.

\subsubsection{Ultra-High Performance Liquid Chromatography-High Definition Mass Spectrometry Analysis}

Methanol extracts were analysed using a Waters Acquity ultra-high performance liquid chromatography coupled in tandem to a Waters SYNAPT G1 quadrupole time-of-flight mass spectrometer (Waters Corporation, Milford, MA, USA). Three technical replicates of each of the four treatments: (1) F. pseudograminearum inoculated, (2) P. alvei amended, (3) primed ( $P$. alvei amended and F. pseudograminearum inoculated) and (4) untreated (control) were performed resulting in 12 injections for each of the nine biological groups [plant tissue (roots, stems and leaves) versus time-point $(1,4$ and 7 d.p.i.)]. Chromatographic separation of the aqueous-methanol extracts was performed using a Waters HSS T3 C18 column $(150 \mathrm{~mm} \times 2.1 \mathrm{~mm} \times 1.8 \mu \mathrm{m})$ thermostatted at $60^{\circ} \mathrm{C}$. Although the T3 column is classified as a C18 reverse phase type, it can separate some polar compounds in addition to the non-polar compounds. The elution gradient was carried out with a binary solvent system consisting of $0.1 \%$ aqueous formic acid (Sigma-Aldrich, Munich, Germany) (solvent A) and $0.1 \%$ formic acid in acetonitrile (Romil Pure Chemistry, Cambridge, UK) (solvent B) at a flow rate of $0.4 \mathrm{~mL} \mathrm{~min}{ }^{-1}$. 
The initial conditions of $98 \% \mathrm{~A}$ and $2 \% \mathrm{~B}$ were held for $13 \mathrm{~min}$ followed by $30 \% \mathrm{~A}$ and $70 \% \mathrm{~B}$ at $14 \mathrm{~min}$. At $15 \mathrm{~min}$, the conditions were changed to 5\% A and 95\% B; these conditions were held for 2 min and then changed to the initial conditions. The analytical column was allowed to equilibrate for $2 \mathrm{~min}$ before the next injection. The total chromatographic run time was $20 \mathrm{~min}$ and the injection volume was $2 \mu \mathrm{L}$. Each sample was analysed in triplicate to account for any analytical variability. The MS detector acquired data in both positive and negative modes following electrospray ionisation. The conditions were set as follows: capillary voltage of $2.5 \mathrm{kV}$, sampling cone at $30 \mathrm{~V}$, extraction cone at $4 \mathrm{~V}$, cone gas flow $50 \mathrm{~L} \mathrm{~h}^{-1}$, desolvation gas flow $550 \mathrm{~L} \mathrm{~h}^{-1}$, source temperature at $120{ }^{\circ} \mathrm{C}$, desolvation temperature at $450{ }^{\circ} \mathrm{C}$, scan time of $0.1 \mathrm{~s}$ and mass range of $100-1000 \mathrm{Da}$. Leucine encephalin (50 pg mL $\left.\mathrm{mL}^{-1}\right) \mathrm{was}^{\mathrm{used}}$ as a calibrant to acquire mass accuracies between 1 and $3 \mathrm{mDa}$ and data were acquired at different collision energies [mass spectrometry at different collision energies (MS ${ }^{\mathrm{E}}$ ) ranging between 10-50 eV] to aid with structural elucidation and annotation of the analytes. Solvent blanks and the QC samples were also analysed in parallel with the sample extracts. The sample acquisition was randomised in the QC sample (six injections) was analysed every 30 injections to monitor and correct changes in the instrument responses. Furthermore, six QC injections were performed in the beginning and end of the batch to insure ensure system equilibration.

\subsubsection{Data Analysis}

In order to visually assess the data, the sets were processed using MarkerlynxXSTM software (Waters Corporation, Milford, USA). Alignment, peak finding, peak integration and retention time (Rt) correction were done on a Rt range of 1.5 and $15 \mathrm{~min}, \mathrm{~m} / \mathrm{z}$ range of $100-1000 \mathrm{Da}$, mass tolerance of $0.05 \mathrm{D}$ and Rt window of $0.2 \mathrm{~min}$. Data were normalised to total intensity (area) using MarkerlynxXS. The datasets thus obtained were exported to the SIMCA (soft independent modelling of class analogy) software version 14 (Umetrics, Umea, Sweden) in order to perform PCA and OPLS-DA. Before performing these multivariate data analyses (MVDA), data were mean centred and Pareto-scaled for both models. The computed and used models were validated as described in the results section. This study mainly focused on the compounds with direct activity against pathogenesis and the associated defence metabolism, thus only the upregulated metabolites were reported on here.

\subsubsection{Metabolite Annotation}

Metabolites were annotated using Taverna workbench (www.taverna.org.uk) for PUTMEDID_LCMS Metabolite ID Workflows [56,57]. The Taverna workflows allow for integrated, automated and high-throughput annotation and putative metabolite identification from LC-ESI-MS metabolomic data. The workflows consist of correlation analysis, metabolic feature annotation and metabolite annotation. A data matrix from MarkerLynx-based data processing was firstly formatted to match the Taverna workbench requirements. Three main workflows formed the Taverna Metabolite ID procedure: (i) Pearson-based correlation analysis (List_CorrData), (ii) metabolic feature annotation (annotate_Massmatch)_allowing for grouping together ion peaks with similar features such as Rt, and annotating features with the type of $\mathrm{m} / \mathrm{z}$ ion (molecular ion, isotope, adduct, others) believed to originate from the same compound. The elemental composition/molecular formula (MF) of each $\mathrm{m} / \mathrm{z}$ ion was then automatically calculated; and (iii) metabolite annotation (matchMF-MF) of the calculated MF (from the output file from workflow 2) was automatically compared and matched to the MF from a pre-defined reference file of metabolites (inhouse library).

For confidence in metabolite annotation, the following steps were performed: (i) the calculated MF of a selected metabolite candidate was manually searched against databases and bioinformatics tools, mainly Chemspider (www.chemspider.com) [58], SorghumbicolorCyc (https://www.plantcyc. org/databases/sorghumbicolorcyc/5.0) [59] and KEGG (Kyoto Encyclopedia of Genes and Genomes, www.genome.jp/kegg/) [60] (ii) structural confirmation through careful inspection of fragmentation patterns by examining the $\mathrm{MS}^{1}$ and $\mathrm{MS}^{\mathrm{E}}$ spectra of the selected metabolite candidate; (iii) comparative 
assessment with/against annotation details of metabolites in S. bicolor, reported in literature [61,62]. Metabolites were annotated to level 2 as classified by the Metabolomics Standard Initiative (MSI) [63].

\subsubsection{Metabolic Pathway Analysis}

Metabolic pathway analysis was performed using the MetPA (Metabolomics Pathway Analysis) component of the MetaboAnalyst bioinformatics tool suite (version 3.0; http://www.metaboanalyst. $\mathrm{ca} /$ ) [64]. This enabled the visualisation of the affected metabolic pathways for the identified metabolites obtained from the OPLS-DA.

\section{Conclusions}

Priming S. bicolor seedlings with P. alvei NAS-6G6 resulted in the induction of systemic resistance against F. pseudograminearum. Results obtain from an untargeted metabolomics approach using an UHPLC-HDMS analytical platform, indicate that the metabolic reprogramming was attributed to an early, enhanced upregulation of phytohormone-, amino acid-, flavonoid-, phenylpropanoid- and lipid metabolism upon inoculation with F. pseudograminearum in primed plants compared with those left naïve. Secondary metabolites/phytoalexins that possess antifungal properties against the genus Fusarium were significantly upregulated in P. alvei-primed versus naïve S. bicolor seedlings. These included epicatechin, hesperetin, coumaroylshikimate, apigenin, naringenin, kaempferol, leucocyanidin, neohesperidin, shikimic acid, caffeic acid, caffeoylquinate and cinnamic acid, giving rise to a significant reduction in crown rot disease severity. These findings were corroborated by the metabolic pathways that were found to be of high significance in the $S$. bicolor response to $P$. alvei-induced priming against F. pseudograminearum. These included glutathione metabolism, shikimate/phenylalanine metabolism and flavonoid biosynthesis. This study revealed strong defence-related metabolic reprogramming in primed sorghum seedlings versus naïve plants as early as 1 d.p.i., pointing to the pre-conditioning of the $P$. alvei-primed plants to quickly halt the invasion and establishment of F. pseudograminearum.

Supplementary Materials: The supplementary material for this article can be found online at http://www.mdpi. com/2218-1989/9/7/150/s1, Figure S1. (A) Microscopic identification of F. pseudograminearum at $400 \times$ magnification. (B) Conidial morphology of F. pseudograminearum taken from Aoki et al. [65]. Figure S2. UHPLC-HDMS BPI chromatograms of ESI-positive data indicating the metabolomic profiles of untreated (black), naïve infected (blue) and primed infected (green) stems obtained at 1 d.p.i. with F. pseudograminearum. Figure S3. UHPLC-HDMS BPI chromatograms of ESI-positive data indicating the metabolomic profiles of untreated (black), naïve infected (blue) and primed infected (green) leaves obtained at 1 d.p.i. with F. pseudograminearum. Figure S4. PCA score/scatter plot of stem samples computed from ESI-positive data. Figure S5. PCA score/scatter plot of leaf samples computed from ESI-positive data. Figure S6. PCA score/scatter plot of root samples computed from ESI-negative data. Figure S7. PCA score/scatter plot of stems samples computed from ESI-negative data. Figure S8. PCA score/scatter plot of leaves samples computed from ESI-negative data. Figure S9. OPLS-DA modelling and variable/feature selection ESI-positive data (stem samples). Figure S10. OPLS-DA modelling and variable/feature selection ESI-positive data (leaf samples). Table S1. Summary of the description and validation of all the generated OPLS-DA models separating naïve versus primed S. bicolor plants. Figure S11. Summary of pathway analysis with MetPA. Figure S12. Venn diagram comparing the number of metabolites shown in Table 2 that were significantly upregulated at 1 d.p.i. (blue), 4 d.p.i. (yellow) and 7 d.p.i. (green) with F. pseudograminearum in primed versus naïve S. bicolor seedlings.

Author Contributions: N.L. and R.C. conceived and guided the project. N.L., I.A.D. and F.T. guided and coordinated the research. R.C. performed the experimental work. P.A.S. did the instrumental analyses of samples. R.C. and F.T. analysed the data and performed the chemometric analyses. All authors contributed to writing and editing of the manuscript. All authors have read and approved the final version of the manuscript.

Funding: This research received no external funding.

Conflicts of Interest: The authors declare no conflict of interest. The funders had no role in the design of the study; in the collection, analyses, or interpretation of data; in the writing of the manuscript, or in the decision to publish the results.

Data availability: The study design information, LC-MS raw data, analyses and data processing information, and the meta-data are being deposited to the EMBL-EBI metabolomics repository-MetaboLights50, with the identifier MTBLS1111 (http://www.ebi.ac.uk/metabolights/MTBLS1111). 


\section{References}

1. Walters, D.; Walsh, D.; Newton, A.; Lyon, G. Induced resistance for plant disease control: Maximizing the efficacy of resistance elicitors. Phytopathology 2005, 95, 1368-1373. [CrossRef] [PubMed]

2. Conrath, U.; Pieterse, C.M.J.; Mauch-Mani, B. Priming in plant-Pathogen interactions. Trends Plant Sci. 2002, 7, 210-216. [CrossRef]

3. Hammerschmidt, R.; Métraux, J.P.; Van Loon, L.C. Inducing resistance: A summary of papers presented at the first international symposium on induced resistance to plant diseases, Corfu, May 2000. Eur. J. Plant Pathol. 2001, 107, 1-6. [CrossRef]

4. Walters, D.; Heil, M. Costs and trade-offs associated with induced resistance. Physiol. Mol. Plant Pathol. 2007, 71, 3-17. [CrossRef]

5. Van Loon, L.C.; Bakker, P.A.H.M.; Pieterse, C.M.J. Systemic resistance induced by rhizosphere bacteria. Annu. Rev. Phytopathol. 1998, 36, 453-483. [CrossRef] [PubMed]

6. Pieterse, C.; Van Wees, S.; Ton, J.; Pelt, J.A.; Van Loon, L.C. Signalling in rhizobacteria-induced systemic resistance in Arabidopsis thaliana. Plant Biol. 2002, 4, 535-544. [CrossRef]

7. Van Peer, R.; Niemann, G.J.; Schippers, B. Induced resistance and phytoalexin accumulation in biological control of Fusarium wilt of carnation by Pseudomonas sp. strain WCS417r. Am. Phytopathol. Soc. 1991, 81, 728-734. [CrossRef]

8. Ongena, M.; Daayf, F.; Jacques, P.; Thonart, P.; Benhamou, N.; Paulitz, T.C.; Bélanger, R.R. Systemic induction of phytoalexins in cucumber in response to treatments with fluorescent pseudomonads. Plant Pathol. 2000, 49, 523-530. [CrossRef]

9. Ramamoorthy, V.; Viswanathan, R.; Raguchander, T.; Prakasam, V.; Samiyappan, R. Induction of systemic resistance by plant growth promoting rhizobacteria in crop plants against pests and diseases. Crop Prot. 2001, 20, 1-11. [CrossRef]

10. Conrath, U. Priming of induced plant defense responses. Adv. Bot. Res. 2009, 51, 361-395.

11. Garcion, C.; Lamotte, O.; Metraux, J.P. Mechanism of defence to pathogens: Biochemistry and physiology. In Induced Resistance for Plant Defence: A Sustainable Approach to Crop Protection; Walters, D., Newton, A., Lyon, G., Eds.; Blackwell Publishing: Oxford, UK, 2007; pp. 109-132. ISBN 9781405134477.

12. Somers, E.; Vanderleyden, J.; Srinivasan, M. Rhizosphere bacterial signalling: A love parade beneath our feet. Crit. Rev. Microbiol. 2004, 30, 205-240. [CrossRef] [PubMed]

13. DeBrito Alvarez, M.; Gagne, S.; Antoun, H. Effect of compost on rhizosphere microflora of the tomato and on the incidence of plant growth-promoting rhizobacteria. Appl. Environ. Microbiol. 1995, 61, 194-199.

14. Bashan, Y.; De-Bashan, L.E. How the plant growth-promoting bacterium Azospirillum promotes plant growth-A critical assessment. In Advances in Agronomy; Sparks, D., Ed.; Elsevier Inc.: Amsterdam, The Netherlands, 2010; Volume 108, pp. 77-136. ISBN 9780123810328.

15. Luz, E.; Hernandez, J.; Bashan, Y.; Maier, R.M. Bacillus pumilus ES4: Candidate plant growth-promoting bacterium to enhance establishment of plants in mine tailings. Environ. Exp. Bot. 2010, 69, 343-352.

16. Ghyselinck, J.; Velivelli, S.L.S.; Heylen, K.; O’Herlihy, E.; Franco, J.; Rojas, M.; De Vos, P.; Prestwich, B.D. Bioprospecting in potato fields in the central Andean highlands: Screening of rhizobacteria for plant growth-promoting properties. Syst. Appl. Microbiol. 2013, 36, 116-127. [CrossRef] [PubMed]

17. Harish, S.; Kavino, M.; Kumar, N.; Saravanakumar, D.; Soorianathasundaram, K.; Samiyappan, R. Biohardening with plant growth promoting rhizosphere and endophytic bacteria induces systemic resistance against banana bunchy top virus. Appl. Soil Ecol. 2008, 39, 187-200. [CrossRef]

18. Walters, D.R. Are plants in the field already induced? Implications for practical disease control. Crop Prot. 2009, 28, 459-465. [CrossRef]

19. Mareya, C.; Tugizimana, F.; Piater, L.; Madala, N.; Steenkamp, P.; Dubery, I. Untargeted metabolomics reveal defensome-related metabolic reprogramming in Sorghum bicolor against infection by Burkholderia andropogonis. Metabolites 2019, 9, 8. [CrossRef]

20. Van Wyk, P.S.; Scholtz, D.S. A selective medium for the isolation of Fusarium spp. from soil debris. Phytophylactica 1995, 18, 67-69.

21. Li, X.; Liu, C.; Chakraborty, S.; Manners, J.M.; Kazan, K. A simple method for the assessment of crown rot disease severity in wheat seedlings inoculated with Fusarium pseudograminearum. J. Phytopathol. 2008, 156, 751-754. [CrossRef] 
22. Tugizimana, F.; Piater, L.; Dubery, I. Plant metabolomics: A new frontier in phytochemical analysis. S. Afr. J. Sci. 2013, 109, 1-11. [CrossRef]

23. Vos, C.M.F.; De Cremer, K.; Cammue, B.P.A.; De Coninck, B. The toolbox of Trichoderma spp. in the biocontrol of Botrytis cinerea disease. Mol. Plant Pathol. 2015, 16, 400-412. [CrossRef] [PubMed]

24. Buensanteai, N.; Yuen, G.Y.; Prathuangwong, S. Priming, signaling, and protein production associated with induced resistance by Bacillus amyloliquefaciens KPS46. World J. Microbiol. Biotechnol. 2009, 25, 1275-1286. [CrossRef]

25. Gutiérrez-Mañero, F.J.; Ramos-Solano, B.; Probanza, A.; Mehouachi, J.; Tadeo, F.R.; Talon, M. The plant-growth-promoting rhizobacteria Bacillus pumilus and Bacillus licheniformis produce high amounts of physiologically active gibberellins. Physiol. Plant. 2001, 111, 206-211. [CrossRef]

26. Bottini, R.; Cassán, F.; Piccoli, P. Gibberellin production by bacteria and its involvement in plant growth promotion and yield increase. Appl. Microbiol. Biotechnol. 2004, 65, 497-503. [CrossRef] [PubMed]

27. Brotman, Y.; Lisec, J.; Meret, M.; Chet, I.; Willmitzer, L.; Viterbo, A. Transcript and metabolite analysis of the Trichoderma-induced systemic resistance response to Pseudomonas syringae in Arabidopsis thaliana. Microbiology 2012, 158, 139-146. [CrossRef] [PubMed]

28. Bennett, R.N.; Wallsgrove, R.M. Secondary metabolites in plant defence mechanisms. New Phytol. 1994, 127, 617-633. [CrossRef]

29. Sharma, P.; Jha, A.B.; Dubey, R.S.; Pessarakli, M. Reactive oxygen species, oxidative damage, and antioxidative defense mechanism in plants under stressful conditions. J. Bot. 2012, 2012, 1-26. [CrossRef]

30. Carrasco, A.; Boudet, A.M.; Marigo, G. Enhanced resistance of tomato plants to Fusarium by controlled stimulation of their natural phenolic production. Physiol. Plant Pathol. 1978, 12, 225-232. [CrossRef]

31. Gunnaiah, R.; Kushalappa, A.C. Metabolomics deciphers the host resistance mechanisms in wheat cultivar Sumai-3, against trichothecene producing and non-producing isolates of Fusarium graminearum. Plant Physiol. Biochem. 2014, 83, 40-50. [CrossRef]

32. Skadhauge, B.; Thomsen, K.K.; Wettstein, D. The role of the barley testa layer and its flavonoid content in resistance to Fusarium infections. Hereditas 2004, 126, 147-160. [CrossRef]

33. Bollina, V.; Kushalappa, A.C.; Choo, T.M.; Dion, Y.; Rioux, S. Identification of metabolites related to mechanisms of resistance in barley against Fusarium graminearum, based on mass spectrometry. Plant Mol. Biol. 2011, 77, 355-370. [CrossRef] [PubMed]

34. Iranshahi, M.; Rezaee, R.; Parhiz, H.; Roohbakhsh, A.; Soltani, F. Protective effects of flavonoids against microbes and toxins: The cases of hesperidin and hesperetin. Life Sci. 2015, 137, 125-132. [CrossRef] [PubMed]

35. Kostyn, K.; Czemplik, M.; Kulma, A.; Bortniczuk, M.; Skała, J.; Szopa, J. Genes of phenylpropanoid pathway are activated in early response to Fusarium attack in flax plants. Plant Sci. 2012, 190, 103-115. [CrossRef] [PubMed]

36. Buśko, M.; Góral, T.; Ostrowska, A.; Matysiak, A.; Walentyn-Góral, D.; Perkowski, J. The effect of Fusarium inoculation and fungicide application on concentrations of flavonoids (apigenin, kaempferol, luteolin, naringenin, quercetin, rutin, vitexin) in winter wheat cultivars. Am. J. Plant Sci. 2014, 5, 3727-3736. [CrossRef]

37. Steinkellner, S.; Mammerler, R. Effect of flavonoids on the development of Fusarium oxysporum f. sp. lycopersici. J. Plant Interact. 2007, 2, 17-23. [CrossRef]

38. Merkl, R.; Hrádková, I.; Filip, V.; Šmidrkal, J. Antimicrobial and antioxidant properties of phenolic acids alkyl esters. Czech J. Food Sci. 2010, 28, 275-279. [CrossRef]

39. Beimen, A.; Witte, L.; Barz, W. Growth characteristics and elicitor-induced reactions of photosynthetically active and heterotrophic cell suspension cultures of Lycopersicon peruvianum (Mill.). Bot. Acta 1992, 105, 152-160. [CrossRef]

40. Kumaraswamy, K.G.; Kushalappa, A.C.; Choo, T.M.; Dion, Y.; Rioux, S. Mass spectrometry based metabolomics to identify potential biomarkers for resistance in barley against Fusarium head blight (Fusarium graminearum). J. Chem. Ecol. 2011, 37, 846-856. [CrossRef]

41. Berkey, R.; Bendigeri, D.; Xiao, S. Sphingolipids and plant defense/disease: The "death" connection and beyond. Front. Plant Sci. 2012, 3, 1-22. [CrossRef]

42. Brodersen, P.; Petersen, M.; Pike, H.M.; Olszak, B.; Skov, S.; Ødum, N.; Jørgensen, L.B.; Brown, R.E.; Mundy, J. Knockout of Arabidopsis accelerated-cell-death11 encoding a sphingosine transfer protein causes activation of programmed cell death and defense. Genes Dev. 2002, 16, 490-502. [CrossRef] 
43. Boubakri, H.; Gargouri, M.; Mliki, A.; Brini, F.; Chong, J.; Jbara, M. Vitamins for enhancing plant resistance. Planta 2016, 244, 529-543. [CrossRef] [PubMed]

44. Asensi-Fabado, M.A.; Munné-Bosch, S. Vitamins in plants: Occurrence, biosynthesis and antioxidant function. Trends Plant Sci. 2010, 15, 582-592. [CrossRef] [PubMed]

45. Nahrstedt, A. Cyanogenic compounds as protecting agents for organisms. Plant Syst. Evol. 1985, 150, 35-47. [CrossRef]

46. Adeyanju, A.; Yu, J.; Little, C.; Rooney, W.; Klein, P.; Burke, J.; Tesso, T. Sorghum RILs segregating for stay-green QTL and leaf dhurrin content show differential reaction to stalk rot diseases. Crop Sci. 2016, 56, 1-9. [CrossRef]

47. Tugizimana, F.; Djami-Tchatchou, A.; Steenkamp, P.A.; Piater, L.A.; Dubery, I.A. Metabolomic analysis of defense-related reprogramming in Sorghum bicolor in response to Colletotrichum sublineolum infection reveals a functional metabolic web of phenylpropanoid and flavonoid pathways. Front. Plant Sci. 2019, 9, 1-20. [CrossRef] [PubMed]

48. Wu, G.; Johnson, S.K.; Bornman, J.F.; Bennett, S.J.; Fang, Z. Changes in whole grain polyphenols and antioxidant activity of six sorghum genotypes under different irrigation treatments. Food Chem. 2017, 214, 199-207. [CrossRef]

49. Hassen, A.I.; Labuschagne, N. Root colonization and growth enhancement in wheat and tomato by rhizobacteria isolated from the rhizoplane of grasses. World J. Microbiol. Biotechnol. 2010, 26, 1837-1846. [CrossRef]

50. Fong, Y.K.; Anuar, S.; Lim, H.P.; Tham, F.Y.; Sanderson, F.R. A modified filter paper technique for long-term preservation of some fungal cultures. Mycologist 2000, 14, 127-130. [CrossRef]

51. Bai, G.-H.; Shaner, G. Variation in Fusarium graminearum and cultivar resistance to Wheat Scab. Plant Dis. 1996, 80, 975-979. [CrossRef]

52. Tugizimana, F. Metabolomic Studies of Induced Defense-Related Changes in Sorghum Bicolor in Response to the Pathogen Colletotrichum Sublineolum. Ph.D. Thesis, University of Johannesburg, Johannesburg, South Africa, 2017.

53. Mitter, V.; Zhang, M.C.; Liu, C.J.; Ghosh, R.; Ghosh, M.; Chakraborty, S. A high-throughput glasshouse bioassay to detect crown rot resistance in wheat germplasm. Plant Pathol. 2006, 55, 433-441. [CrossRef]

54. Xi, K.; Turkington, T.K.; Chen, M.H. Systemic stem infection by Fusarium species in barley and wheat. Can. J. Plant Pathol. 2008, 30, 588-594. [CrossRef]

55. Akinsanmi, O.A.; Mitter, V.; Simpfendorfer, S.; Backhouse, D.; Chakraborty, S. Identity and pathogenicity of Fusarium spp. isolated from wheat fields in Queensland and northern New South Wales. Aust. J. Agric. Res. 2004, 55, 97-107. [CrossRef]

56. Brown, M.; Dunn, W.B.; Dobson, P.; Patel, Y.; Winder, C.L.; Francis-Mcintyre, S.; Begley, P.; Carroll, K.; Broadhurst, D.; Tseng, A.; et al. Mass spectrometry tools and metabolite-specific databases for molecular identification in metabolomics. Analyst 2009, 134, 1322-1332. [CrossRef] [PubMed]

57. Brown, M.; Wedge, D.C.; Goodacre, R.; Kell, D.B.; Baker, P.N.; Kenny, L.C.; Mamas, M.A.; Neyses, L.; Dunn, W.B. Automated workflows for accurate mass-based putative metabolite identification in LC/MS-derived metabolomic datasets. Bioinformatics 2011, 27, 1108-1112. [CrossRef] [PubMed]

58. Chemspider. Available online: http://www.chemspider.com (accessed on 10 June 2018).

59. SorghumbicolorCyc. Available online: http://www.plantcyc.org (accessed on 30 September 2018).

60. Kyoto Encyclopedia of Genes and Genomes. Available online: http://www.kegg.jp (accessed on 5 June 2018).

61. Dykes, L.; Rooney, L.W. Sorghum and millet phenols and antioxidants. J. Cereal Sci. 2006, 44, 236-251. [CrossRef]

62. Kang, J.; Price, W.E.; Ashton, J.; Tapsell, L.C.; Johnson, S. Identification and characterization of phenolic compounds in hydromethanolic extracts of sorghum wholegrains by LC-ESI-MS ${ }^{n}$. Food Chem. 2016, 211, 215-226. [CrossRef] [PubMed]

63. Sumner, L.W.; Samuel, T.; Noble, R.; Gmbh, S.D.; Barrett, D.; Beale, M.H.; Hardy, N. Proposed minimum reporting standards for chemical analysis Chemical Analysis Working Group (CAWG) Metabolomics Standards Initiative (MSI). Metabolomics 2007, 3, 211-221. [CrossRef] [PubMed]

64. MetaboAnalyst. Available online: http://www.metaboanalyst.ca (accessed on 15 October 2018).

65. Aoki, T.; O’Donnell, K. Morphological characterization of Gibberella coronicola sp. nov., obtained through mating experiments of Fusarium pseudograminearum. Mycoscience 1999, 40, 443-453. [CrossRef] 